\title{
ISRT Lecture
}

\section{Towards improved clinical and physiological assessments of recovery in spinal cord injury: a clinical initiative}

\author{
PH Ellaway*, ${ }^{*}$ P Anand ${ }^{1}$, EMK Bergstrom ${ }^{2}$, M Catley ${ }^{1}$, NJ Davey ${ }^{1}$, HL Frankel ${ }^{2}$, A Jamous ${ }^{2}$, C Mathias ${ }^{1}$, \\ A Nicotra ${ }^{1}$, G Savic $^{2}$, D Short ${ }^{3}$ and S Theodorou ${ }^{1}$ \\ ${ }^{1}$ Division of Neuroscience and Psychological Medicine, Imperial College, London; UK; ${ }^{2}$ National Spinal Injuries Centre, \\ Stoke Mandeville Hospital, Aylesbury, UK; ${ }^{3}$ Midlands Centre for Spinal Injuries, Oswestry, UK
}

\begin{abstract}
Clinical practice and scientific research may soon lead to treatments designed to repair spinal cord injury. Repair is likely to be partial in the first trials, extending only one or two segments below the original injury. Furthermore, treatments that are becoming available are likely to be applied to the thoracic spinal cord to minimise loss of function resulting from damage to surviving connections. These provisos have prompted research into the improvement of clinical and physiological tests designed (1) to determine the level and density of a spinal cord injury, (2) to provide reliable monitoring of recovery over one or two spinal cord segments, and (3) to provide indices of function provided by thoracic spinal root innervation, presently largely ignored in assessment of spinal cord injury. This article reviews progress of the Clinical Initiative, sponsored by the International Spinal Research Trust, to advance the clinical and physiological tests of sensory, motor and autonomic function needed to achieve these aims. Spinal Cord (2004) 42, 325-337. doi:10.1038/sj.sc.3101596; Published online 17 February 2004
\end{abstract}

Keywords: spinal cord injury; outcome measures; ASIA classification; assessment; recovery

\section{Introduction}

There is currently no medical or surgical treatment for complete traumatic spinal cord lesion. In the United Kingdom, spinal cord injury (SCI) is not recognised as a priority by the National Health Service despite the number of sufferers; SCI affects approximately 60 out of every 100000 of the population, with 700 new cases each year. ${ }^{1,2}$ The last $10-15$ years have however seen basic scientific research in animals identify several procedures that offer realistic promise for the treatment of SCI (for a review, see Fawcett ${ }^{3}$ ). Treatment leading to recovery of significant function will require a multifaceted approach. This may include manipulation of nerve growth and inhibitory factors, insertion of bridging grafts, and the deployment of cell types (eg olfactory ensheathing cells, differentiated stem cells) that facilitate the growth of neuronal axons or replace neurons. With advances already having been made in animal studies in these fields, it is anticipated that clinical trials of treatments for paralysis caused by spinal cord injury in man will start within a few years. The trials will most probably involve subjects with a mid-thoracic injury

*Correspondence: PH Ellaway, Imperial College, Charing Cross Campus, St Dunstan's Road, London W6 8RP, UK

The International Spinal Research Trust lecture, International Spinal Cord Society, Beijing, 15-18 October 2003 since such interventions are likely to cause only minimal further functional deterioration in the event that the treatment is unsuccessful or damages surviving connections to spinal roots. The consequence of treatment causing further damage to the spinal cord in persons with cervical injury is likely to be a serious impediment to immediate treatment of most SCI sufferers. The risk of even a minor upward shift in the clinical neurological level of a cervical lesion cannot afford to be taken since the outcome could be loss of finger, hand or arm movements (C5-T1) or even the ability to breath spontaneously (C3-C5). Although treatment applied to the thoracic spinal cord would be safer, it is likely to result in only very modest improvement in function. Regeneration of axons in rat spinal cord has progressed for only a short distance beyond the caudal boundary of a lesion. ${ }^{4,5}$ If the first attempts at treatment in man result in regenerating axons reaching target neurons sited only one or two cord segments below the caudal boundary of the original lesion, the recovery of function will be limited and may represent no real increase in the quality of life. However, the experimental trials will be worthwhile as a test-bed for future treatment at a cervical level. At the cervical level, regaining function over a short distance will provide significant functional benefits. The problem facing the first trials of treatment 
applied to the thoracic spinal cord is that the anticipated limited recovery of function will be difficult to quantify. The physiology of sensory, motor, and autonomic systems in the trunk has been studied less than in the limbs. The present clinical neurological assessment criteria largely ignore muscles innervated by thoracic spinal cord segments. Also, they usually do not include tests of cardiac, vasomotor, and sudomotor functions. Additionally, it is likely that successful recovery of function below a lesion will not include those segments actually at the site of damage since spinal cord neurons in the grey matter at that level will most probably have been destroyed. New improved tests of function will therefore need to distinguish subtle differences in density of spinal cord lesions above and below the injury site.

In 2001, the International Spinal Research Trust (ISRT) commissioned a Clinical Initiative with the aim of developing a wide range of improved clinical and physiological tests for determining the boundaries and density of SCI, and for reliable monitoring of recovery over one or two spinal cord segments. The damage caused by spinal cord injury is not completed at the instant of an accident. Secondary changes may occur and even in patients with complete SCI it takes several months before the neurological impairment is stable. The ISRT Clinical Initiative is therefore undertaking both baseline and longitudinal studies of SCI sufferers to test developing clinical and physiological assessment techniques.

The work is being carried out by four groups clinical, motor, sensory, and autonomic - working closely together. Whenever possible, patient volunteers are seen by all four groups. If this is not feasible, then at least a detailed clinical neurological examination is performed on all patients undergoing any physiological studies, so that the correlation between tests can be established upon completion of data collection.

\section{Clinical neurological component}

At present, the American Spinal Injury Association (ASIA) standards are internationally agreed standards for neurological classification of SCI. ${ }^{6}$ The fifth revision is currently in use. ${ }^{7}$ There is a clear need to improve the existing clinical neurological assessment of SCI patients and to develop new clinical tests for determining the level and density of spinal cord lesions. In particular, there are no tests for determining the motor level of lesions to the thoracic spinal cord. There is also a perceived need to assess the inter-rater reliability for classifying SCI using International Standards. ${ }^{8,9}$

\section{Clinical motor tests}

Inter-rater reliability of clinical motor tests (manual muscle testing according to ASIA/IMSOP standards and dynamometry testing of the 20 ASIA key muscles) has been analysed in 30 patients with SCI. The results of two examiners (a senior physiotherapist and a clinical scientist) obtained on the same patient within $48 \mathrm{~h}$ were compared. The individual muscle testing of the 20 ASIA key muscles showed strong agreement for manual muscle testing, and strong correlation for dynamometry measurements between the two examiners. Dynamometry adds precision to standard manual muscle testing for muscle grades 3 and 4. This initial study, showing a high level of agreement between two experienced examiners, has been extended to include clinical sensory testing and is being conducted on a larger number of patients. This will establish the extent to which the degree of variability in final results is due to inter-rater differences in clinical testing.

\section{Clinical sensory tests}

In order to reduce the inter-rater and test-retest variability, additional photographic documentation of sensory levels on the trunk has been added to the standard clinical sensory testing. The exact levels of the last normal dermatome and the extent of any zone of partial preservation are marked bilaterally on the abdomen and back. It is anticipated that photographic images will reduce the risk that an independent clinical rater might misjudge the location and extent of a dermatome determined by a previous rater. The addition of photographic evidence will also assist in longitudinal repeatability studies.

\section{Cutaneous electrical perceptual sensory threshold}

In addition to the possible limitations in the assessment of SCI that are imposed by inter-rater variability, the neurological measurement and monitoring of somatosensory function according to the guidelines of the ASIA lacks resolution. ${ }^{6}$ Attempts have been made to improve the quantitative nature of somatosensory tests using the perceptual threshold to electrical stimulation. $^{10-13}$ In an extensive study of spinal dermatomes between $\mathrm{C} 3$ and L3 in control subjects, the perceptual threshold to low-frequency $(3 \mathrm{~Hz})$ electrical stimulation of the skin was found not to correlate with two-point discriminative ability. ${ }^{14}$ There was however, good correlation between results from equivalent dermatomes on the left and right sides for both measures. In a repeatability study in trunk dermatomes, the results correlated better for electrical perceptual threshold than for two-point discrimination. ${ }^{15}$

As part of developing quantitative sensory tests for monitoring changes in the level and density of spinal cord lesions, we have now established normative values for perceptual threshold to cutaneous electrical stimulation in control subjects. Perceptual thresholds were measured in 18 control subjects at ASIA sensory key points for dermatomes $\mathrm{C} 3-\mathrm{S} 2$ bilaterally. Perceptual threshold was defined as the lowest ascending stimulus intensity $(\mathrm{mA})$ at which the subject reported sensation. The results may be conveniently plotted as the mean perceptual threshold plus two standard deviations superimposed on a diagram of the vertebral column (Figure 1). The plot provides a visual template for 
a

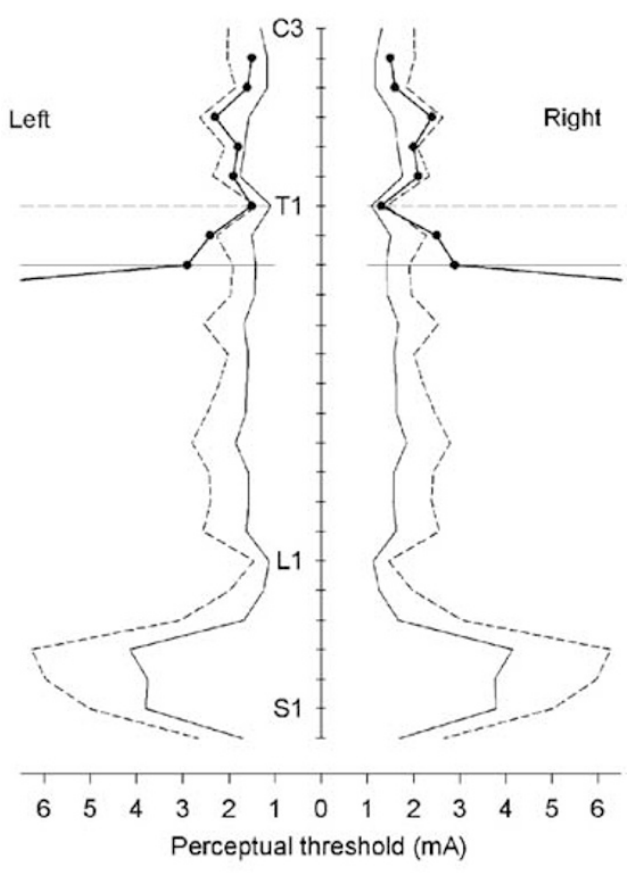

b

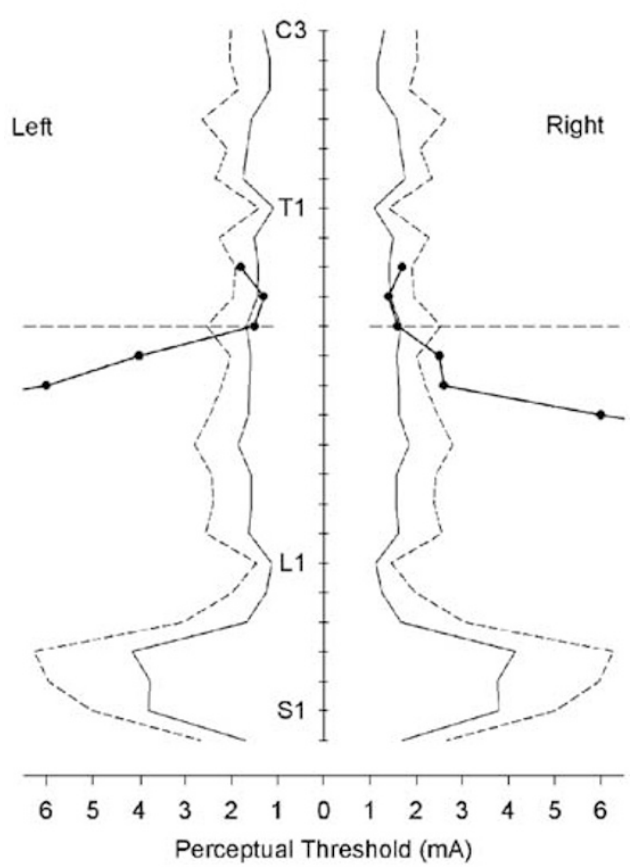

Figure 1 Cutaneous electrical perceptual thresholds at different spinal segmental levels for two patients with complete (a) and incomplete (b) SCI plotted with reference to normative values. Each plot consists of threshold determinations in $\mathrm{mA}$ (filled symbols) superimposed on the mean (solid line) plus two SD (dashed line) values for 18 control subjects. The lowest clinically determined normal level in each case is represented by a horizontal dashed line. A horizontal solid line indicates the lower boundary of a zone of partial preservation in the patient with complete SCI. Perceptual threshold was not reached using the maximum current $(10 \mathrm{~mA})$ in the incomplete patient at levels $\mathrm{T} 9$ and below comparing results from subjects with SCI. When perceptual thresholds for a complete (T1, ASIA grade A) SCI patient are superimposed on the normal template (Figure 1a), the level of SCI can be established as the lowest spinal segment for which the patient's perceptual threshold is within the control group range $($ mean +2 standard deviation (SD)). A zone of partial preservation at T2-3 is clearly indicated by thresholds exceeding normative values plus two SDs. In an incomplete (T5, ASIA grade D) SCI patient (Figure 1b), thresholds are normal above the level of lesion and raised below the lesion. In addition, the test reveals asymmetry of impairment of sensory function below the lesion. In other patients, electrical perceptual threshold tests have revealed raised sensory thresholds at levels rated normal during routine clinical assessment.

Perceptual threshold tests have been carried out on 26 patients. Preliminary results suggest that perceptual threshold may be more sensitive to discrete sensory changes than standard clinical examination, and that it could be useful for determining both the level of SCI and, in patients with incomplete injury, the density of SCI.

\section{Vibration perceptual threshold}

Similarly, vibration threshold was measured in 12 control subjects at bony prominences within ASIA dermatomes $\mathrm{C} 3-\mathrm{S} 1$ bilaterally and over spinous processes C7-L5. Vibration at $100 \mathrm{~Hz}$ frequency was applied via a hand-held probe (Bio-thesiometer, Biomedical Instrument Co., Newbury, OH, USA). Vibration threshold was recorded as the lowest ascending vibration amplitude in microns at which the subject reported sensation. The study has confirmed the simplicity and reproducibility of the vibration test in control subjects. Its usefulness in quantifying sensory impairment in spinal cord injury is currently being established. Preliminary results in 18 patients with SCI suggest that vibration threshold may be useful in assessing the level and particularly the density of SCI below injury level.

\section{Disability measures}

Disability measures, which assess a patient's functional independence, have been added to the clinical testing protocol. Together with the standard disability instrument, Functional Independence Measure (FIM) ${ }^{16}$ and two new functional measures, Spinal Cord Independence Measure (SCIM) ${ }^{17}$ and Walking Index for Spinal Cord Injury (WISCI) ${ }^{18,19}$ are being assessed in patients with SCI during initial rehabilitation as part of two international studies.

\section{Motor component}

The central neural control of skeletal muscle by the corticospinal tract and by reflex afferents is reasonably well documented and understood for limbs but not for trunk muscles in man. Since the first interventive 
treatments for SCI are likely to involve subjects with thoracic injury, the Clinical Initiative investigation of motor function has concentrated on myotomes innervated from the thoracic spinal cord. The Initiative has also had to recognise that neural control of musculature in SCI is affected by plasticity of CNS function in addition to the loss of descending inputs to motoneurones. Physiological ${ }^{20,21}$ and imaging studies ${ }^{22,23}$ lend support to the notion that central neural plasticity may be induced by SCI. The study of these plastic changes to the control of musculature is of importance with regard to the recovery of function following treatment. Any regeneration of descending axons to segments below a lesion will need to re-establish connection with spinal interneurones and motoneurones. However, the patterns of activity in those regenerated axons will have been altered by the plastic changes resulting from the original injury. Supraspinal and propriospinal networks controlling the target muscles of regenerating axons neurones may require the original plastic changes to be reversed to restore normal function. The assessment of motor function will therefore need to be able to recognise any change in function altered as a result of plasticity, and to monitor any reversal. In particular, any reversal may be a significant, if indirect, pointer to the re-establishment of more normal functional connectivity during recovery.

\section{Corticospinal innervation of limb muscles}

There are several aspects of the corticospinal drive to muscles below the site of incomplete SCI that are altered by a lesion. Threshold and latency of motor evoked potentials (MEPs) in response to transcranial magnetic stimulation (TMS) of the motor cortex are increased, ${ }^{24,20}$ probably as a result of reduced numbers of corticospinal axons reaching the motoneurone pool. Cortical inhibition, as revealed by the reduction in voluntary EMG to low-intensity TMS ${ }^{25}$ has a higher threshold and an increased latency, suggesting a downregulation of cortical inhibitory circuitry. ${ }^{20}$ The recruitment of MEPs in thenar muscles to increasing strength of TMS of the motor cortex, or with increasing voluntary effort using a fixed strength TMS, is less steep in stable incomplete cervical SCI subjects at rest than in normal subjects. ${ }^{23}$ These altered patterns of recruitment of thenar muscles in SCI are more similar to the normal situation for muscles of the leg. Since the leg muscles have a less evident corticospinal innervation than muscles of the hand, the finding for the thenar muscles in SCI presumably reflects a reduced corticospinal innervation resulting from the lesion. In a longitudinal study of incomplete SCI, the raised TMS thresholds for MEPs at rest and during facilitation with voluntary effort, the raised threshold for cortical inhibition, and the increased latencies of MEPs and suppressed EMG (inhibition) were all found to be constant from the time of the earliest measurements following injury and for up to 3 years. ${ }^{21}$ This contrasted with clear signs of functional recovery as measured by neurological sensory and motor scores, particularly during the 3-6 months following injury. It was postulated that the functional differences in corticospinal control revealed by TMS might be a prerequisite for natural recovery processes that progress over months following injury.

\section{Corticospinal innervation of erector spinae muscles}

TMS of the motor cortex and surface electromyography (EMG), both of which are noninvasive techniques, have been used to examine the corticospinal innervation of contralateral erector spinae muscles in control subjects ${ }^{26}$ and in patients with complete mid-thoracic (T4-T7) spinal cord injury. ${ }^{27,28}$ Contrary to a previous finding, ${ }^{29}$ MEP responses could be recorded from muscles at segments below the lesion. MEPs elicited in myotomes below the level of clinically complete lesions occur presumably because individual heads of the erector spinae muscles span several segments. In comparison with control subjects, the threshold TMS required to evoke an MEP was actually lower above the

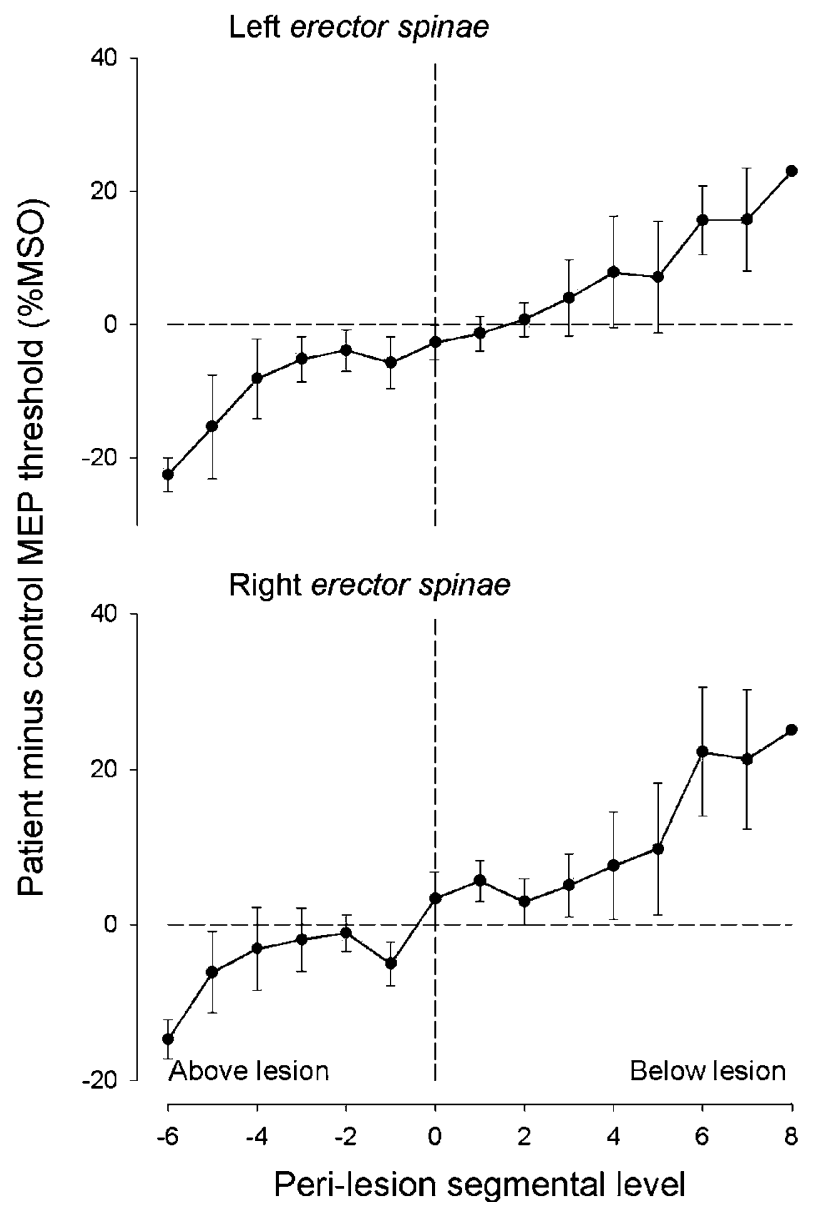

Figure 2 Thresholds for eliciting MEPs in paravertebral muscles by TMS of the motor cortex in patients with complete mid-thoracic (T4-T7) SCI. The difference (means \pm SE) between patients and controls of the average MEP thresholds is expressed as a percentage of maximum stimulator output (\%MSO) for the thoracic segments. Note that the patients have been aligned with respect to the level of their lesion 
lesion, presumably as a result of central plasticity. In contrast, the threshold was raised below the lesion and increased in a linear fashion rostro-caudally (Figure 2), most likely reflecting the absence of direct segmental innervation requiring greater input from collateral innervation above the lesion. The crossover from lower to higher than normal threshold coincided with the level of lesion. This feature of the responses to TMS indicates that the technique may be useful for monitoring recovery. It is reasonable to anticipate that regeneration and recovery of function might be reflected in a caudal shift of the pattern of TMS threshold values.

Uncrossed cortical innervation of erector spinae muscles During quiet standing, when an arm is abducted there is activation of contralateral trunk muscles to maintain stability. The manoeuvre is used routinely in stroke rehabilitation physiotherapy. In man, ipsilateral proximal arm muscles and axial muscles may be accessed via uncrossed pathways from the motor cortex $^{30,31}$ and such routes may be more prominent after CNS injury, for example, in stroke. ${ }^{32,33} \mathrm{We}$ have studied corticospinal facilitation of contralateral back muscles (erector spinae) during arm abduction in normal subjects in anticipation that the technique may provide a tool for the assessment of motor function controlled by uncrossed corticospinal or other descending pathways. The rationale is that the various descending motor tracts are located in different parts of the spinal cord and might be affected differently by traumatic spinal cord injury. MEP responses to TMS of the motor cortex during arm abduction were facilitated in the contralateral but not the ipsilateral erector spinae muscles, and this was evident in supine as well as standing positions. ${ }^{34,35} \mathrm{We}$ argued that the drive to the contralateral erector spinae muscles is corticospinal and likely to be ipsilateral in its cortical origin. The study is now being extended to patients with incomplete thoracic spinal injury. Persistence or otherwise of contralateral facilitation during arm abduction and a comparison with the crossed corticospinal innervation of the erector spinae (see above) will allow any differential impact of the injury on these spinal cord pathways to be assessed.

Corticospinal innervation of internal oblique muscles The muscles used in swallowing have hemispheric asymmetry of representation in the motor cortex, with dominant control from one cortex. ${ }^{36}$ Axial muscles are also known to have a greater proportion of uncrossed ipsilateral corticospinal drive than muscles of the limbs (see above). The ability to assess the function of the anterior (uncrossed) corticospinal tract in SCI would be of considerable value when establishing motor recovery. To this effect, we have investigated the relative dominance of the corticospinal drive to the left and right internal oblique muscles of the lower abdomen. ${ }^{37}$ Using TMS to evoke MEPs in first dorsal interosseus, deltoid, and internal oblique muscles, we found ipsilat- eral MEPs to be over twice as prevalent and twice as large for the internal oblique compared with the shoulder and hand muscles. The ratio of ipsilateral to contralateral responses was compared for each cortex. The mean $( \pm \mathrm{SE})$ value of the larger of the two ratios was $1.14 \pm 0.28$ compared with $0.27 \pm 0.04$, indicating substantial dominance by one hemisphere. Latencies of the ipsilateral MEPs were 3-5 ms longer than contralateral responses. The longer latency is consistent with transmission through ipsilateral corticospinal tracts. ${ }^{31,32}$ Our preliminary results indicate that one cortex can exert a dominant control over internal oblique muscles on both sides of the body, suggesting heavy involvement of uncrossed pathways.

\section{Corticospinal innervation of intercostal muscles}

Electromyographic responses of intercostal muscles to transcranial magnetic stimulation (TMS) have been used to evaluate patients with cervical myelopathy. ${ }^{38} \mathrm{We}$ have extended the technique to examine the pattern of facilitation with changing voluntary effort at six intercostal muscle levels in control subjects. ${ }^{39}$ In nine healthy volunteers TMS was delivered using a 9-cm circular coil centred over the vertex with the induced current flowing clockwise. Surface electrodes were placed in each intercostal space on the right side of the body at approximately 2 and $4 \mathrm{~cm}$ from the sternal edge. Recordings were made from the first intercostal space (T6/T7) up to the mid-clavicular line (T1/T2). Subjects breathed through a tube with a slow leak connected to a pressure meter that provided feedback of expiratory effort. The size of MEPs at all recording sites became larger with increasing voluntary expiratory effort. Averaging across all subjects, a progressive facilitation was observed on increasing the voluntary effort of expiration. The amount of facilitation was proportionally greater at low levels of effort (5-10\% MEE). This is similar to the pattern for hand muscles. ${ }^{23}$ However, the pattern of facilitation of MEPs with increasing effort is less steep in hand muscles of patients with incomplete SCI. We are now examining intercostal muscles in spinal cord-injured patients to see whether the pattern of facilitation changes in the manner observed for hand muscles. Such a diagnostic feature will assist in determining the impact of cord injury on muscles with thoracic innervation. One patient with a clinically complete lesion at T5 is illustrated in Figure 3a. Unlike control subjects, there is relatively little facilitation with weak expiratory effort (5 and 10\% MEE) at any level, including sites well above the lesion. This may reflect plastic reorganization of the surviving corticospinal input to the intercostals, given that the lesion will have denervated the most caudal motoneurones. It is also evident that MEPs are recorded below the clinical level of the lesion, which may indicate a zone of partial preservation. Cortically evoked inhibition of voluntary activity in hand muscles is reduced in incomplete SCI and the suppression of EMG in response to TMS has increased latency. ${ }^{20,40}$ Figure $3 b$ shows that the latency 

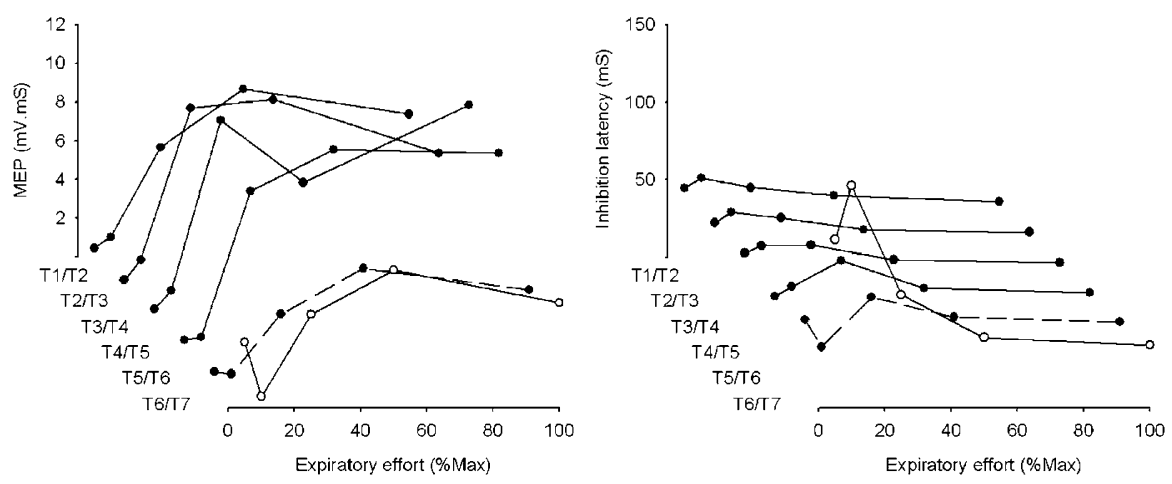

Figure 3 Facilitation of MEP responses and latency of cortically evoked inhibition in intercostal muscles in a patient with spinal cord injury (T5. ASIA grade A). Left: size of the MEP, recorded at intercostal spaces T1/T2-T6/T7, plotted against percentage expiratory effort. Right: latency of the suppression of EMG in response to TMS, at a strength below threshold for eliciting a MEP, plotted against percentage expiratory effort. Solid lines and solid symbols - above lesion. Dashed line and solid symbols - at the level of lesion. Solid line and open symbols - below lesion

of cortical inhibition of intercostal muscle EMG is fairly constant at different levels of voluntary effort above the lesion in the spinal cord injured patient. However, below the lesion (T6/T7), the latency is markedly prolonged at weak levels of voluntary effort, a pattern that is typical of hand muscles in patients with incomplete lesions. This again suggests a zone of partial preservation of motor function that was not detected by clinical neurological assessment. These preliminary results suggest that noninvasive TMS and EMG recording from intercostal muscles may provide diagnostic measures of the level and density of thoracic SCIs.

Short- and long-latency reflexes in erector spinae muscles The few reports concerning spinal reflex activity of human trunk muscles identify both short- and longlatency EMG responses to tapping muscles or spinous processes. ${ }^{41-45}$. The short-latency component is an oligosynaptic or monosynaptic stretch reflex. The longlatency component appears to involve a supraspinal, possibly transcortical, route. There are several factors that make it difficult to be sure of the true nature and extent of these reflex connections. One problem is that paraspinal muscles may be innervated from more than one spinal segment and muscle fibres may span several segments. This can account for EMG responses being recorded below the level of a spinal cord lesion in response to stimulation above the lesion. ${ }^{46,28}$ Second, the interpretation of complex EMG responses is problematical since longer latency responses may be confused with ongoing modulation of EMG caused by an early component. In an attempt to clarify these issues, we have conducted studies into the reflex responses of paraspinal muscles in response to tapping muscle and spinous processes in normal control subjects $^{47}$ and subjects with complete SCI at a thoracic level. An electromagnetic device provided a brief mechanical stimulus to the spinal processes or adjacent muscles of the thoracic spine. Short- and long-latency reflexes were recorded in control subjects. The shortlatency reflex was more readily elicited by prodding muscle than the vertebral column, and was rarely recorded more than one segment away from the site of stimulation. The long-latency component was more readily elicited by prodding the spinous processes, and could be recorded from myotomes throughout the thoraco-lumbar spinal cord, irrespective of the level of stimulation. The origin, distribution, and latency of the short-latency reflex have the characteristics of the monosynaptic stretch reflex elicited by muscle spindle afferents. The long-latency reflex is probably excited by stimulation of mechanoreceptors within the intraspinous ligaments. ${ }^{48}$ Its distribution and long latency are compatible with a supraspinal pathway. ${ }^{49}$ Our initial results from 11 clinically complete thoracic (T1-T12) SCI patients further support the concept of these two reflexes having separate origins and pathways. Figure 4 compares results from an SCI patient with a complete lesion at T7 (ASIA grade A) with those of a control subject. The SCI patient shows short-latency responses to both muscle and spinous process stimuli at the level of stimulation, but no long-latency reflex at any level even though the stimuli were administered above the level of lesion. Stimuli below the lesion in this patient again elicited only segmental short-latency responses. This pattern was typical of all 11 SCI patients. The reason why long-latency responses are lacking in SCI is unclear. If propriospinal neurones are involved in the pathway then disruption of axons by the lesion may be a contributing factor. Whatever the reason, mechanically evoked reflexes in paraspinal muscles appear to show promise as tools for determining the level and completeness of SCI. Importantly, the reflexes involve only noninvasive methodology.

\section{Sensory component}

As with the motor system, sensory testing in SCI is presently directed mainly at the limbs, although the 


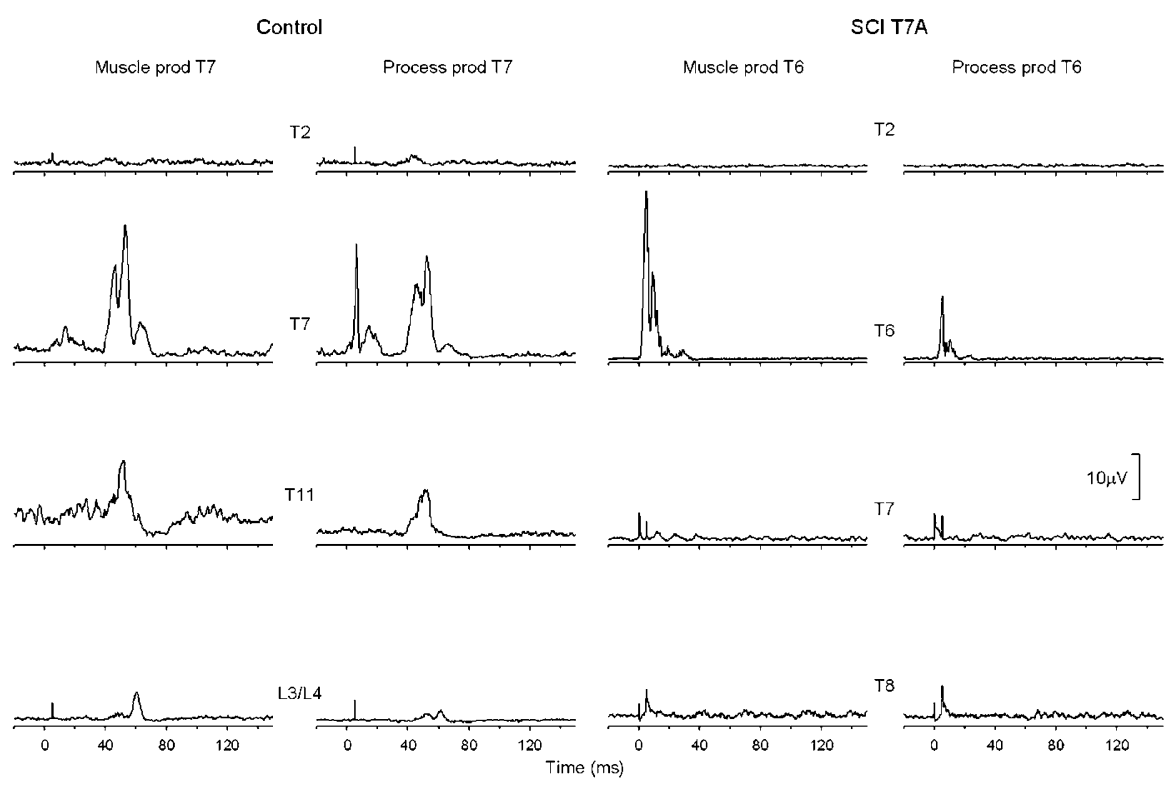

Figure 4 Reflex responses in erector spinae muscles at different segmental levels to mechanical stimulation (prod) of the paraspinal muscle or spinal process at a specific level. Left: control subject - prod at T7. Right: a complete (T7, ASIA A) SCI patient - prod at T6. The traces are averages of full-wave-rectified EMG recordings. Prod stimuli of 5 ms duration were applied at $0 \mathrm{~ms}$, (note the stimulus artefacts of variable magnitude). Short-latency reflex responses, starting during the stimulus and lasting up to $20 \mathrm{~ms}$, can be seen at the site of stimulation in both the control subject and SCI patient (second row from top). They are not apparent at other segments. Long-latency responses (approximately $40-60 \mathrm{~ms}$ ) are elicited only in the control subject, are most evident at the site of stimulation (T7), and can be seen at all the four recording sites

thoracic dermatomes are well defined and may readily be assessed. Currently, neurological sensory testing usually involves only a limited number of modalities, such as light touch and pinprick and, occasionally, thermal threshold. Quantitative sensory testing (QST) is regarded as a potentially useful tool for measuring sensory impairment in neurological conditions including $\mathrm{SCI},{ }^{50}$ provided repeated measures are taken ${ }^{51}$ and other criteria are included. ${ }^{52}$ We have therefore employed a wide range of sensory tests and added axon-flare responses, elicited by histamine injection or heat provocation, to the gamut of QST. In addition, we have begun a sensory repeatability study designed to assess the utility of QST in SCI.

The axon-flare responses included in our QST investigation clearly involve both sensory and autonomic nervous system components. In a broader approach to developing tools for assessment of autonomic function, sympathetic skin responses (SSR) to a variety of stimuli have been examined in SCI patients, together with the indices of cardiac and vasomotor function.

\section{Quantitative sensory testing (QST)}

A total of 50 patients with complete or incomplete SCI according to the ASIA impairment scale (International Standards for Neurological classification of Spinal Cord
Injury, Revised 2000) were studied. The following tests were employed:

- Light touch threshold using Semmes-Weinstein monofilaments (A Ainsworth, University College London, UK). The number of the hair detected with the lowest force determined the threshold. An abnormal value was taken as any value greater than the number 3 filament.

- Vibration perception threshold measured with a biothesiometer (Biomedical Instrument Co., Newbury, OH, USA). Abnormal measures for the index finger and great toe were taken as any value greater than $10 \mathrm{~V} .^{53,54}$

- Thermal thresholds to cold perception (CPT), warm perception (WPT), cold pain (CP), and heat pain (HP) were measured with the Medoc Thermal Sensory Analyser (TSA-2001, Israel). An abnormal value was taken as any value greater than 2SD from control average values.

- Pain experienced in the absence of overt stimulation was assessed by direct interview using a visual analogue scale (VAS) rating pain on a scale from 0 (= no pain) to 10 (= worse imaginable pain), at rest and on movement, and the McGill Pain Questionnaire. ${ }^{55,56}$

- Cutaneous sensory axon reflex was evaluated using laser Doppler fluxmetry (PF4 Perimed, Stockholm, Sweden) and tracing the area of the flare in response 

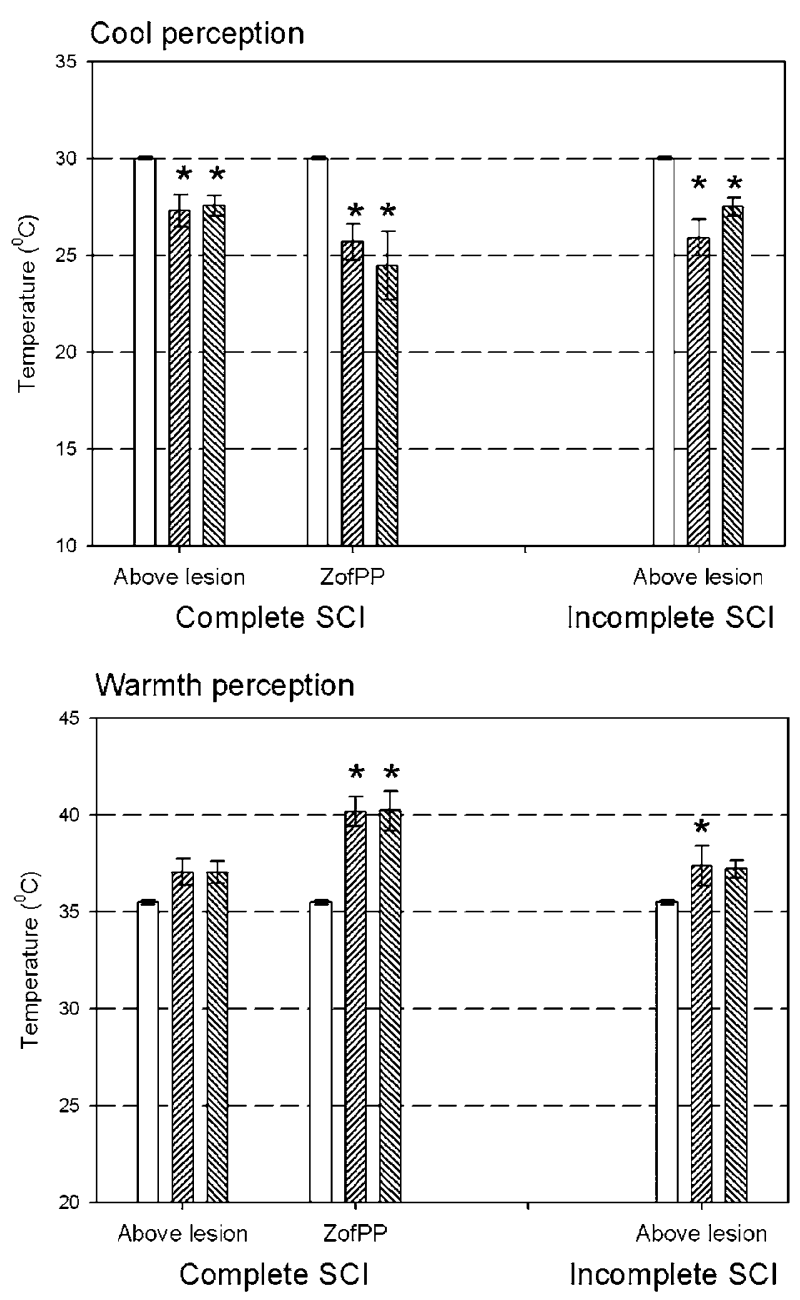

Figure $5 \mathrm{Cool}$ and warmth perception thresholds. Mean $( \pm \mathrm{SE})$ thresholds for 10 control subjects (open bars), 34 complete, and 16 incomplete SCI patients tested on the left (rising hatch) and right (falling hatch) dermatomes. ZofPP zone of partial preservation. Asterisks indicate statistically significant $(P<0.05)$ differences from control values

to intradermal histamine injection $(0.03 \mathrm{ml}$ of $1 \mathrm{mg} / \mathrm{ml}$ histamine acid phosphate - Martindale Pharmaceuticals, UK).

In a group of 34 patients with complete injury, QST was carried out for dermatomes at the neurological level of the lesion, that is, the most caudal segment of the spinal cord with normal sensory and motor function on both sides of the body. QST was also applied to the zone of partial preservation (ZPP), namely those dermatomes caudal to the neurological level that remain partially innervated. In a second group of 16 patients with incomplete injury, QST was again applied at the neurological level of the lesion and at a number of dermatomes below the level of injury on both sides of the body. The rationale for not extending QST above the neurological level of lesion is that our previous (unpublished) studies in the laboratory have shown no differences in sensory thresholds several dermatomes above the clinical sensory level in patients with complete SCI.

In complete injury above the level of lesion, no significant deviations (Mann-Whitney $t$-test) from normal $(n=10)$ were found in monofilament threshold, vibration threshold or thermal threshold to warmth perception. There was, however, a significant increase in cool perception threshold (Figure 5a). Cold pain and heat pain thresholds were not significantly different from normal. In the zone of partial preservation, monofilament threshold and the thermal thresholds for both cool and warmth perception were all raised. In addition, some patients reported a misinterpretation of sensation (cold for warm or vice versa), or an undefined sensation rather than thermal. Thermal allodynia was also observed in eight subjects. Below the zone of partial preservation, there was a total sensory loss, as judged by the limits of tests.

In incomplete injury above the level of lesion, no deviations from normal were found in monofilament or vibration threshold. There was, however a significant difference in cool perception and warmth perception threshold (Figure 5b), both being raised compared with controls. Below the level of lesion, monofilament, vibration, cool, and warm perception threshold were all raised and there was misinterpretation of thermal modalities. Thermal allodynia was noticed in four subjects.

In all, 12 complete and four incomplete SCI patients reported spontaneous pain at and/or below the level of lesion. The mean VAS score in the complete SCI patients was 4.2 (moderate pain), and in the incomplete lesions was 2.6 (mild pain). The McGill pain questionnaire showed that the pain was related to sensory components, whereas the other categories (eg affective) were not as prominent.

\section{Sensory repeatability study}

Five complete SCI patients have been assessed on two occasions as part of a preliminary repeatability study. The average time between injury and the first test was 37 weeks and between the first and the second assessment was 43 weeks. Above the level of lesion, no significant changes were observed in vibration, thermal, cold or heat perception thresholds between the first and the second assessment. Monofilament threshold to light touch was lower on the second visit. The most consistent findings, however, were the changes to thermal pain appreciation. In all the five patients, there was either a marked increase in threshold or no appreciation of pain to cold as low as $0^{\circ} \mathrm{C}$ on one or both sides on the second assessment. There were also significant elevations in heat pain thresholds from one assessment to the next. Since cold and heat pain thresholds showed marked changes at two time points at varied and relatively long intervals after injury, it appears that evoked pain thresholds may vary more than detection perception thresholds. The alterations in thermal pain threshold may indicate differential changes to the spinothalamic nociceptive 
pathway close to the site of lesion that are not reflected in other ascending tracts. In the zone of partial preservation, there were no significant changes in monofilament, vibration or thermal thresholds between the first and second visit. Below the level of lesion, there remained a total sensory loss.

In five incomplete SCI patients, there were no significant changes in any sensory modalities above or below the level of lesion between the first and second visit (average 27 weeks separation). Only one incomplete patient with neurogenic pain participated in the repeatability study, reporting increased pain (VAS at rest rated 10 compared to 8.4 in the first visit). In another incomplete patient, a clear change over three segments in the last normal sensory level was observed by the same investigator (from T12 D to L3 D).

\section{Histamine induced axon-reflex flare responses}

Tests have been conducted in patients with complete SCI. ${ }^{57}$ Axon-reflex vasodilation, as measured by laser doppler (flux), in response to subcutaneous injection of histamine, was present in all patients above and below the lesion, but was significantly diminished below the level of lesion, by an average $39 \%$ of control values. The flux increase was also significantly correlated with area of the flare. Histamine-induced axon-reflex flare responses would appear to provide a practical means of determining the level of SCI with respect to sensory and autonomic function.

\section{Conclusions concerning QST}

QST detected abnormal, raised thermal perception thresholds in dermatomes clinically defined as normal (last normal sensory level) with ASIA. The technique is therefore more sensitive than the standard clinical examination, and has been shown to be a repeatable means of testing. QST offers a range of values compared to the standard clinical examination, and should improve the detection of natural recovery or of small changes after intervention. Skin-flare responses are significantly reduced below the level of a lesion. Although not providing a robust measure of spinal cord function, the test would appear to be a useful adjunct to other QST procedures.

\section{Autonomic component}

The following noninvasive methods have been used to assess autonomic function:

- determination of sympathetic cholinergic activity using the sympathetic skin response (SSR) to electrical and physiological (sound, voluntary gasp \& bladder tap) stimuli;

- continuous measurement of blood pressure and heart rate and derived cardiac and vasomotor parameters at rest and as responses to stimuli during SSR tests (Portapres II, TNO-TPD Biomedical Instruments, Amsterdam);
- determination of local cutaneous vasomotor activity using the axon-reflex vasodilation to heat provocation (thermostatic laser-Doppler probe, PF4, Perimed, Stockholm, Sweden).

\section{Sympathetic skin response}

Previous reports ${ }^{58,59}$ suggested that SSRs might be elicited by appropriate stimuli when the thoracolumbar spinal cord is isolated from supraspinal structures in SCI. In our study ${ }^{60}$ of 17 patients with complete cord section (C3-L1), SSRs in response to electrical nerve, stimulation were consistently absent below the level of lesion. A palmar response could be obtained to stimulation of the median nerve, provided the lesion was at or below T6. One exception was a complete SCI patient, assessed neurologically as T2 grade A on the ASIA scale, in whom a reduced palmar response was recorded. This suggests either that simply one or two segments (T1-T2) are sufficient to support the response or that the lesion was incomplete in respect of autonomic function. A further 21 patients with SCI have now been studied using physiological stimuli to elicit SSRs. The stimuli included an auditory click, a voluntary gasp, and a supra-pubic mechanical tap. Table 1 summarises the results from all patients. In patients with complete injury at levels C4-T5, weak $(<50 \%$ control) palmar responses could be elicited in four out of six and plantar responses in two out of six patients. Figure 6 shows recordings from a complete SCI patient with a C7 lesion. Both palmar and plantar SSRs, with a delay of approximately $2 \mathrm{~s}$, are evident in response to gasps and bladder taps but not to sound clicks. In seven further SCI patients with complete lesions from T6 to T11, palmar SSRs were observed more frequently and occasionally with near normal amplitudes, in good agreement with our previous study. ${ }^{61}$ The only plantar SSRs were observed in a T11 complete patient. Eight further SCI patients with incomplete lesions were studied. The presence and amplitude of palmar and plantar SSRs in response to physiological stimuli did not appear to correlate with the neurological severity of the lesion, as judged by the ASIA rating. Furthermore, one incomplete patient (C4) had no responses to any of the physiological stimuli despite an ASIA grade C. We conclude that the presence of SSR is likely to reflect preserved descending autonomic pathways in the spinal cord in patients classified by ASIA as motor and sensory complete. Equally, a classification of sensory or motor incomplete cannot be taken to indicate the status of descending autonomic pathways.

\section{Cardiovascular assessment - systemic}

A variety of haemodynamic parameters were studied together with the SSR at rest and in response to different stimuli. These included systolic and diastolic blood pressure, heart rate, stroke volume, cardiac output, ejection fraction, and total peripheral resistance. The 
Table 1 Presence and amplitude of palmar and plantar SSR to auditory, inspiratory gasp and suprapubic bladder tapping stimuli in subjects with SCI

\begin{tabular}{|c|c|c|c|c|c|c|c|}
\hline \multirow{2}{*}{ Subject no. } & \multirow{2}{*}{ Level \& ASIA grade } & \multicolumn{3}{|c|}{$\mathrm{R} / \mathrm{L}$ Palmar } & \multicolumn{3}{|c|}{$\mathrm{R} / \mathrm{L}$ Plantar } \\
\hline & & AS & G & BT & AS & $\mathrm{G}$ & BT \\
\hline 1 & $\mathrm{C} 4 \mathrm{~A}$ & $-1-$ & $-1-$ & $-1-$ & $-1-$ & $-1-$ & $-1-$ \\
\hline 2 & C7 A & $-1-$ & $+/+$ & $+1-$ & - & $+/+$ & $+1-$ \\
\hline 3 & T1 A & $-1-$ & $-1-$ & $-1-$ & $-1-$ & $-1-$ & $-1-$ \\
\hline 4 & $\mathrm{~T} 2 \mathrm{~A}$ & $+1+$ & $+/+$ & $+/+$ & $-1-$ & $-1-$ & $-1-$ \\
\hline 5 & $\mathrm{~T} 3 \mathrm{~A}$ & $-1+$ & $-1+$ & $-1-$ & $-1-$ & $-1+$ & $-1-$ \\
\hline 6 & T4 A & $+/++$ & $++/++$ & $\mathrm{np}$ & $-1-$ & $-1-$ & $\mathrm{np}$ \\
\hline 7 & T6 A & $-1-$ & $-1-$ & $-1-$ & $-1-$ & $-1-$ & $-1-$ \\
\hline 8 & T6 A & $++/++$ & $+/+$ & $-1-$ & $-1-$ & $-1-$ & $-1-$ \\
\hline 9 & T7 A & $+/++$ & $+/+$ & np & $-1-$ & $-1-$ & $\mathrm{np}$ \\
\hline 10 & T8 A & $+/++$ & $++/++$ & $+/+$ & $-1-$ & $-1-$ & $-1-$ \\
\hline 11 & T8 A & $+/+$ & $+/+$ & $-1-$ & $-1-$ & $-1-$ & $-1-$ \\
\hline 12 & T9 A & $-1-$ & $+/+$ & $-1-$ & $-1-$ & $-1-$ & $-1-$ \\
\hline 13 & $\mathrm{~T} 11 \mathrm{~A}$ & $++/++$ & $++/++$ & $++/++$ & $++/++$ & $++/++$ & $++/++$ \\
\hline 14 & T9 B & $++/++$ & $++/++$ & $++/++$ & $++/++$ & $++/++$ & $-1-$ \\
\hline 15 & $\mathrm{C} 4 \mathrm{C}$ & $-1-$ & $-1-$ & $-1-$ & $-1-$ & $-1-$ & $-1-$ \\
\hline 16 & $\mathrm{C} 7 \mathrm{C}$ & $-1-$ & $++/+$ & $-1-$ & $-1-$ & $-1-$ & $-1-$ \\
\hline 17 & L1 C & $++/++$ & $++/++$ & $++/++$ & $++/++$ & $++/++$ & $+/++$ \\
\hline 18 & L3 C & $++/++$ & $++/++$ & $++/++$ & $++/+$ & $++/+$ & $+1+$ \\
\hline 19 & L4 C & $++/++$ & $++/++$ & $++/++$ & $-1-$ & $+/+$ & $++/+$ \\
\hline 20 & C4 D & $-1-$ & $++/++$ & $+1+$ & $-1-$ & $++/++$ & $+/+$ \\
\hline 21 & T12 D & $+/+$ & $+/+$ & $+/+$ & $-1-$ & $+/+$ & $-1-$ \\
\hline
\end{tabular}

AS, auditory stimulus; -, SSR absent;

$\mathrm{G}=$ inspiratory gasp; + , SSR amplitude $<50 \%$ of normal mean;

$\mathrm{BT}=$ bladder tapping; ++ , SSR amplitude $>50 \%$ of normal mean; np, not performed

physiological stimuli used to elicit SSRs caused no significant changes in either control subjects, complete or incomplete SCI patients. We conclude from this negative result that the stimuli were insufficient to provoke sympathetic adrenergic changes sufficient to alter cardiac function or systemic haemodynamics.

In a preliminary study, four further SCI patients with complete injury (C4, C7, T4, and T6) have been studied using a variety of stimuli expected to elicit cardiovascular responses. Basal supine blood pressure and heart rate were similar in SCI patients and control subjects. Head-up tilt caused falls in blood pressure and increases in heart rate in the patients with cervical injury. The Valsalva manoeuvre showed only small changes in blood pressure, but this test was compromised by the difficulties experienced by SCI subjects in maintaining intrathoracic pressure. Pressor stimuli above the level of lesion (eg mental arithmetic) had no effect compared with control subjects where a modest rise in blood pressure occurred. Stimuli below the level of lesion (ice pack, bladder tap) that are known to cause an exaggerated rise in blood pressure leading to autonomic dysreflexia were ineffective. The combination of tilt table testing with other measures of systemic sympathetic activation and parasympathetic cardiac effects should provide overall measures of systemic vascular and cardiac autonomic control. ${ }^{62}$ Note that caution is required with the use of a tilt-table in regard to postural hypotension. ${ }^{63}$

Cardiovascular assessment - site specific

Skin blood flow increases in response to local heating reaching an initial peak within $10 \mathrm{~min}$, followed by a progressive further rise to a plateau lasting longer. The initial peak in heat-provoked vasodilatation is considered to be mediated by a sensory axon reflex and the longer plateau by the local release of nitric oxide. Using histamine injection, SCI patients were found to have diminished axon reflexes below the level of injury. ${ }^{57}$ Since heat provocation provides a more acceptable physiological stimulus than histamine, our aim was to establish whether the former would provide a reliable test of the patency of the axon reflex in SCI. Four complete (T6-T9) and five incomplete (C4-L4) SCI patients were studied. There was no significant difference in resting skin blood flow or the initial peak response to heat provocation on the hand in SCI compared with normal control subjects. However, the peak response on the foot was significantly lower in complete SCI. The reduced vasodilatation below the level of a complete SCI may indicate a disturbance of 
a

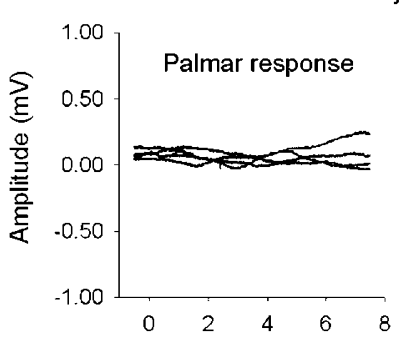

b

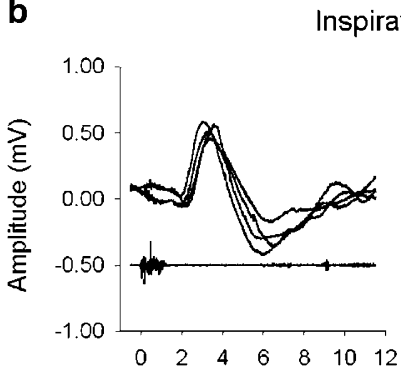

Inspiratory gasp
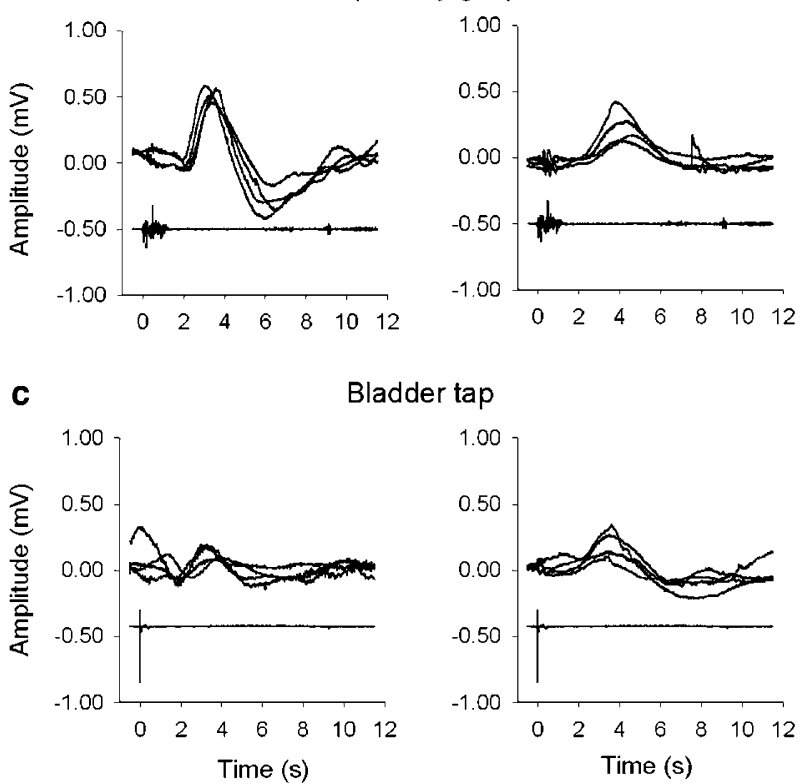

Bladder tap

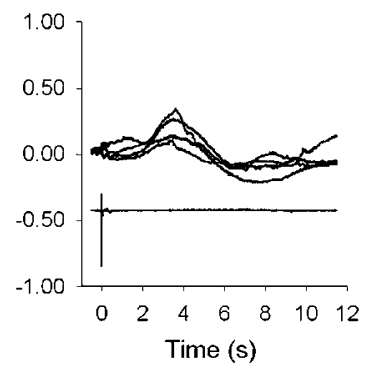

Figure 6 Sympathetic skin responses to physiological stimuli in a complete SCI patient with a C7 lesion (patient 2 in Table 1). Four right-sided palmar or plantar recordings are superimposed in response to auditory (a), inspiratory gasp (b), and bladder tap (c) stimuli. Sympathetic skin responses with a latency of approximately $2 \mathrm{~s}$ are evident to inspiratory gasp and bladder tap, but not to the auditory stimuli. Responses to inspiratory gasp and bladder tap were triggered by EMG signals (inset) recorded from the sternocleidomastoid and rectus abdominis muscles, respectively

the axon reflex or altered sympathetic vasomotor control. The result appears to provide a noninvasive means of evaluating localised sympathetic adrenergic control and a further means of determining the integrity of descending spinal autonomic pathways.

\section{General conclusions}

This article has reviewed the progress made by the Clinical Initiative into improving methods for detecting change and recovery in SCI in man. The Initiative, sponsored by the International Spinal Research Trust, is incomplete at this time. However, a number of advances in techniques for the assessment of SCI have emerged. Clearly, the usefulness of any suite of assessment tools will depend not only on the merits of the individual components, but also on the practicality of administer- ing several tests to the one patient. A large number of tests should not present a problem if an individual is cooperative and the anticipated benefit from treatment is an adequate incentive. The Clinical Initiative arose from the anticipation that interventive remedial treatment for the repair of SCI will materialise in the next 510 years. In the first trials, it is anticipated that recovery may be limited to one or two spinal segments. Remedial intervention will most probably be offered to patients with thoracic injury, since treatment of the thoracic spinal cord is least likely to provoke increased disability. Thus, improved techniques necessary for monitoring recovery (or deterioration) over as little as one segment will clearly need to be more elaborate and time consuming than present tests. Furthermore, if the injury is to the thoracic spinal cord, the clinical and physiological tests will need to target organs innervated by those segments. This Initiative has identified a number of tools that are showing promise in meeting these aims. Bearing in mind also the need to restrict the duration of assessment to a minimum compatible with the durability of the patient, the following limited set of tests appears to be the most appropriate recommendation at this stage in the Clinical Initiative:

- conventional clinical, neurological assessment;

- dynamometry for appropriate muscles;

- motor evoked potential responses to transcranial magnetic stimulation;

- reflexes in paraspinal muscles;

- quantitative sensory testing including electrical sensory perceptual threshold;

- sympathetic skin response.

Although tailored to assess change in the thoracic segments, this protocol (with the exception of reflex testing of specific back muscles) is broadly applicable to injury at other segmental levels.

\section{Acknowledgements}

The Clinical Initiative is funded by The International Spinal Research Trust. We thank the patients and clinical staff at the National Spinal Injuries Centre, Stoke Mandeville Hospital for their generous participation and P Kirkwood for expert advice.

\section{References}

1 Smith M. 'Making a Difference'. Efficacy of Specialist versus Non-specialist Management of Spinal Cord Injury. Spinal Injuries Association: London, 1999.

2 Bird R, Kuhn G. Bed numbers. Spinal Columns 2001; 1: 3.

3 Fawcett J. Repair of spinal cord injuries: where are we, where are we going? Spinal Cord 2002; 40: 615-623.

4 Kunkel-Bagden E, Dai HN, Bregman BS. Recovery of function after spinal cord hemi-section in newborn and adult rats: differential effects on reflex and locomotor function. Exp Neurol 1992; 116: 40-51.

5 Raineteau O, Fouad K, Bareyre FM, Schwab ME. Reorganization of descending motor tracts in the rat spinal cord. Eur J Neurosci 2002; 16: 1761-1771. 
6 Maynard Jr FM, Bracken MB, Creasey G, Gitunno Jr JF, Donovan WH, Ducker TB et al. International Standards for Neurological and Functional Classification of Spinal Cord Injury. American Spinal Injury Association. Spinal Cord 1997; 35: 266-274.

7 Marino RJ (ed). International Standards for Neurological Classification of Spinal Cord injury, 5th edn. American Spinal Injury Association: Chicago, 2000.

8 Donovan WH, Brown DJ, Ditunno Jr JF, Dollfus P, Frankel HL. Neurological issues. Spinal Cord 1997; 35: 275-281.

9 Cohen ME, Ditunno Jr JF, Donovan WH, Maynard Jr FM. A test of the 1992 International Standards for Neurological and Functional Classification of Spinal Cord Injury. Spinal Cord 1998; 36: 554-560.

10 Rendell MS, Dovgan DJ, Bergman TF, O'Donnell GP, Drobny EP, Katims JJ. Mapping diabetic sensory neuropathy by current perception threshold testing. Diabetes Care 1989; 12: 636-640.

11 Pitei DL, Watkins PJ, Stevens MJ, Edmonds ME. The value of the neurometer in assessing diabetic neuropathy by measurement of the current perception threshold. Diabet Med 1994; 11: 872-876.

12 Donaghue VM, Giurini JM, Rosenblum BI, Weissman PN, Veves A. Variability in function measurements of three sensory foot nerves in neuropathic diabetic patients. Diabetes Res Clin Pract 1995; 29: 37-42.

13 Kozarski J, Panajtovic LJ, Novakovic M, Stosic S, Bojovic M. Use of Bernard's diadynamic current in evaluating pain sensitivity in transplanted microvascular flaps. Vojnosanti Pregled 1999; 56: 599-605.

14 Davey NJ, Nowicky AV, Zaman R. Somatopy of perceptual threshold to cutaneous electrical stimulation in man. Exp Physiol 2001; 86: 127-130.

15 Yewlett AD, Tufail SR, Davey NJ. Somatotopy of the human trunk to cutaneous electrical stimulation and twopoint discrimination. J Physiol 2003; 547P: 81P.

16 Uniform Data System for Medical Rehabilitation. Guide for the Uniform Data Set for Medical Rehabilitation (Adult FIM), Version 4.0. State University of New York at Buffalo: Buffalo, NY, 1993.

17 Catz A, Itzkovich M, Steinberg F, Philo O, Ring H, Ronen J et al. The Catz-Itzkovich SCIM: a revised version of The Spinal Cord Independence Measure. Disabil Rehabilit 2001; 23: 263-268.

18 Ditunno JF, Ditunno PL, Graziani V, Scivoletto G, Bernardi M, Castellano V et al. Walking index for spinal cord injury (WISCI): an international multicentre validity and reliability study. Spinal Cord 2000; 38: 234-243.

19 Ditunno PL, Ditunno JF. Walking index for spinal cord injury (WISCI II): scale revision. Spinal Cord 2001; 39: 654-656.

20 Davey NJ, Smith HC, Wells E, Maskill DW, Savic G, Ellaway $\mathrm{PH}$ et al. Responses of thenar muscles to transcranial magnetic stimulation of the motor cortex in incomplete spinal cord injury patients. J Neurol Neurosurg Psychiatry 1998; 65: 80-87.

21 Smith HC, Savic G, Frankel HL, Jamous MA, Ellaway $\mathrm{PH}$, Maskill DW et al. Neurological assessment and corticospinal function studied over time following incomplete spinal cord injury. Spinal Cord 2000; 38: 292-300.

22 Puri BK, Smith HC, Cox IJ, Sargentoni J, Savic G, Maskill DW et al. The human motor cortex after incomplete spinal cord injury: an investigation using proton magnetic resonance spectroscopy. J Neurol Neurosurg Psychiatry 1998; 65: 748-754.

23 Davey NJ, Smith HC, Maskill DW, Savic G, Ellaway PH. Comparison of input-output patterns in the corticospinal system of normal subjects and incomplete spinal cord injured patients. Exp Brain Res 1999; 127: 382-390.

24 Brouwer B, Bugaresti J, Ashby P. Changes in corticospinal facilitation of lower limb spinal motor neurons after spinal cord lesions. J Neurol Neurosurg Psychiatry 1992; 55: 20-24.

25 Davey NJ, Romaiguère $\mathrm{P}$, Maskill DW, Ellaway $\mathrm{PH}$. Suppression of voluntary motor activity revealed using transcranial magnetic stimulation of the motor cortex in man. J Physiol 1994; 477: 223-235.

26 Nowicky AV, McGregor AH, Davey NJ. Corticospinal facilitation studied during voluntary contraction of human back muscles. Motor Control 2001; 3: 270-280.

27 Cariga P, Catley M, Sahal A, Savic G, Ellaway PH, Davey NJ. Corticospinal activation of paraspinal muscles above and below complete thoracic spinal cord injury in man. Abstr Soc Neurosci 2001; 27: 304-308.

28 Cariga P, Catley M, Nowicky AV, Savic G, Ellaway PH, Davey NJ. Segmental recording of cortical motor evoked potentials from thoracic paravertebral myotomes in complete spinal cord injury. Spine 2002; 27: 1438-1443.

29 Ertekin C, Uludag B, On A, Yetimalar Y, Ertas M, Colakoglu $\mathrm{Z}$ et al. Motor-evoked potentials from various levels of paravertebral muscles in normal subjects and in patients with focal lesions of the spinal cord. Spine 1998; 23: $1016-1022$.

30 Wassermann EM, Fuhr P, Cohen LG, Hallett M. Effects of transcranial magnetic stimulation on ipsilateral muscles. Neurology 1991; 41: 1795-1799.

31 Ziemann U, Ishii K, Borgheresi A, Yaseen Z, Battaglia F, Hallett $\mathrm{M}$ et al. Dissociation of the pathways mediating ipsilateral and contralateral motor-evoked potentials in human hand and arm muscles. J Physiol 1999; 518: 895-906.

32 Netz J, Lammers T, Homberg V. Reorganisation of motor output in the non-affected hemisphere after stroke. Brain 1997; 120: 1579-1586.

33 Alagona G, Delvaux V, Gerard P, De Pasqua V, Pennisi G, Delwaide PJ et al. Ipsilateral motor responses to focal transcranial magnetic stimulation in healthy subjects and acute-stroke patients. Stroke 2001; 32: 1304-1309.

34 Lisle R, Loxton-Edwards B, McGregor AH, Nowicky AV, Davey NJ. Corticospinal facilitation of contralateral back muscles during arm abduction in man. J Physiol 2001; 531P: 139P.

35 Davey NJ, Lisle R, Loxton-Edwards B, McGregor AH, Nowicky AV. Activation of back muscles during abduction of the contralateral arm in man. Spine 2002; 27: 1355-1360.

36 Hamdy S, Aziz Q, Rothwell JC, Singh KD, Barlow J, Hughes DG et al. The cortical topography of human swallowing musculature in health and disease. Nat Med 1996; 2: 1217-1224.

37 Strutton PH, Beith I, Theodorou S, Catley M, McGregor AH, Davey NJ. Corticospinal activation of internal oblique muscles has a strong ipsilateral component and can be lateralised. Abstr Soc Neurosci 2003; 28: 77.12.

38 Misawa T, Ebara S, Kamimura M, Tateiwa Y, Kinoshita T, Takaoka K. Evaluation of thoracic myelopathy by transcranial magnetic stimulation. J Spinal Disord 2001; 14: 439-444. 
39 Theodorou S, Catley M, Strutton PH, Davey NJ. Examination of intercostals muscle facilitation evoked by transcranial magnetic stimulation (TMS) in man. $J$ Physiol 2003; 547P: C144.

40 Shimizu T, Hino T, Komori T, Hirai S. Loss of the muscle silent period evoked by transcranial magnetic stimulation of the motor cortex in patients with cervical cord lesions. Neurosci Lett 2000; 286: 199-202.

41 Dimitrijevic MR, Gregoric MR, Sherwood AM, Spencer WA. Reflex responses of paraspinal muscles to tapping. J Neurol Neurosurg Psychiatry 1980; 43: 1112-1118.

42 Kondo T, Bishop B, Shaw CF. Phasic stretch reflex of the abdominal muscles. Exp Neurol 1986; 94: 120-140.

43 Tani T, Yamamoto H, Ichimiya M, Kimura J. Reflexes evoked in human erector spinae muscles by tapping during voluntary activity. Electroencephalogr Clin Neurophysiol 1997; 105: 194-200.

44 Zedka M, Prochazka A, Knight B, Gillard D, Gauthier M. Voluntary and reflex control of human back muscles during induced pain. J Physiol 1999; 520: 591-604.

45 Myriknas SE, Beith ID, Harrison PJ. Stretch reflexes in the rectus abdominis muscle in man. Exp Physiol 2000; 85: 445-450.

46 Gough JG, Koepke GH. Electromyographic determination of motor root levels in erector spinae muscles. Arch Phys Med Rehabil 1966; 47: 9-11.

47 Strutton PH, McGregor AH, Catley M, Davey NJ. Mechanically-evoked long and short latency reflexes in human erector spinae muscles. J Physiol 2002; 543P: 88P.

48 Solomonow M, Zhou BH, Harris M, Lu Y, Baratta RV. The ligamento-muscular stabilizing system of the spine. Spine 1998; 23: 2552-2562.

49 Toshikazu T, Yamamoto H, Ichimiya M, Kimura J. Reflexes evoked in human erector spinae muscles by tapping during voluntary activity. Electroencephalogr Clin Neurophysiol 1997; 105: 194-200.

50 Hayes KC, Wolfe DL, Hsieh JT, Potter PJ, Krassioukov A, Durham CE. Clinical and electrophysiologic correlates of quantitative sensory testing in patients with incomplete spinal cord injury. Arch Phys Med Rehabil 2002; 83: 1612-1619.

51 Krassioukov A, Wolfe DL, Hsieh JT, Hayes KC, Durham CE. Quantitative sensory testing in patients with incomplete spinal cord injury. Arch Phys Med Rehabil 1999; 80: 1258-1263.

52 Shy ME, Frohman EM, So YT, Arezzo JC, Cornblath DR, Giuliani MJ et al. Quantitative sensory testing: report of the Therapeutics and Technology. Assessment Subcommittee of the American Academy of Neurology. Neurology 2003; 60: 898-904.

53 Bloom S, Till S, Sonksen P, Smith S. Use of a biothesiometer to measure individual vibration thresholds and their variation in 519 non-diabetic subjects. $\mathrm{Br}$ Med $\mathrm{J}$ (Clin Res Ed) 1984; 288: 1793-1795.

54 Guy RJ, Clark CA, Malcolm PN, Watkins PJ. Evaluation of thermal and vibration sensation in diabetic neuropathy. Diabetologia 1985; 28: 131-137.

55 Melzack R. The McGill pain questionnaire: major properties and scoring methods. Pain 1975; 1: 277-299.

56 Price D, McGrath P, Refii A. The validation of visual analogue scales as ratio measures for chronic and experimental pain. Pain 1983; 17: 45-56.

57 Kuesgen B, Frankel HL, Anand P. Decreased cutaneous sensory axon-reflex vasodilation below the level of lesion in patients with complete spinal cord injury. Somatosensory Motor Res 2002; 19: 149-152.

58 Fuhrer MJ. Skin conductance responses mediated by the transected human spinal cord. J Appl Physiol 1971; 30: 663-669.

59 Fuhrer MJ. Effects of stimulus site on the pattern of skin conductance responses evoked from spinal man. J Neurol Neurosurg Psychiatry 1975; 38: 749-755.

60 Cariga P, Catley M, Mathias CJ, Savic G, Frankel HL, Ellaway PH. Organisation of the sympathetic skin response in spinal cord injury. J Neurol Neurosurg Psychiatry 2002; 72: $356-360$.

61 Cariga P, Catley M, Savic G, Frankel HL, Mathias CJ, Ellaway $\mathrm{PH}$. The sympathetic skin response in complete human spinal cord injury. J Physiol 2001; 533P: 68-69.

62 Mathias CJ, Frankel HL. Autonomic disturbances in spinal cord lesions. In: Bannister R, Mathias CJ (eds). Autonomic Failure. Oxford Press: Oxford, 2002, Chapter 51, pp 494-513.

63 El Masry WS. Physiological instability of the spinal cord following injury. Paraplegia 1993; 31: 273-275. 